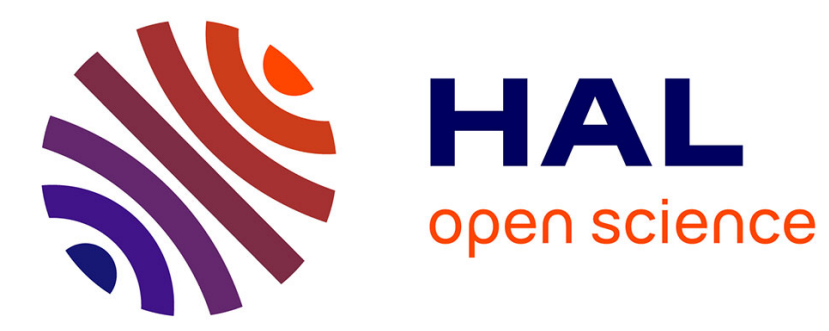

\title{
Synthesizing Accurate Floating-Point Formulas
}

\author{
Arnault Ioualalen, Matthieu Martel
}

\section{To cite this version:}

Arnault Ioualalen, Matthieu Martel. Synthesizing Accurate Floating-Point Formulas. ASAP: Application-Specific Systems, Architectures and Processors, Jun 2013, Washington, DC, United States. pp.113-116, 10.1109/ASAP.2013.6567563 . hal-00835736

\section{HAL Id: hal-00835736 https://hal.science/hal-00835736}

Submitted on 21 Jun 2013

HAL is a multi-disciplinary open access archive for the deposit and dissemination of scientific research documents, whether they are published or not. The documents may come from teaching and research institutions in France or abroad, or from public or private research centers.
L'archive ouverte pluridisciplinaire HAL, est destinée au dépôt et à la diffusion de documents scientifiques de niveau recherche, publiés ou non, émanant des établissements d'enseignement et de recherche français ou étrangers, des laboratoires publics ou privés. 


\title{
Synthesizing Accurate Floating-Point Formulas ${ }^{\dagger}$
}

\author{
Arnault Ioualalen ${ }^{1,2}$ and Matthieu Martel ${ }^{1,2}$ \\ ${ }^{1}$ Univ. Perpignan Via Domitia, Digits, Architectures et Logiciels Informatiques, F-66860, Perpignan, France \\ ${ }^{2}$ Univ. Montpellier II, CNRS, LIRMM, UMR 5506, F-34095, Montpellier, France \\ Email: \{arnault.ioualalen, matthieu.martel\}@univ-perp.fr
}

\begin{abstract}
Many critical embedded systems perform floatingpoint computations yet their accuracy is difficult to assert and strongly depends on how formulas are written in programs. In this article, we focus on the synthesis of accurate formulas mathematically equal to the original formulas occurring in source codes. In general, an expression may be rewritten in many ways. To avoid any combinatorial explosion, we use an intermediate representation, called APEG, enabling us to represent many equivalent expressions in the same structure. In this article, we specifically address the problem of selecting an accurate formula among all the expressions of an APEG. To validate our approach, we present experimental results showing how APEGs, combined with profitability analysis, make it possible to significantly improve the accuracy of floating-point computations.
\end{abstract}

\section{INTRODUCTION}

Most critical control systems of recent planes, spatial vehicles or power-plants rely on floating-point computations [1] yet this arithmetic is not intuitive [11]. Indeed, it is very difficult to predict by hand the accuracy of the evaluation of a formula, given certain ranges for the inputs and, recently, static analysis techniques have been developed to infer safe error bounds for the computations arising in critical programs written in C [6], [7]. However these techniques do not indicate how to improve the accuracy if the inferred error bounds are not satisfying.

Our work concerns the synthesis at compile-time of accurate formulas, for given input ranges, to replace the expressions written by the programmers in source codes [10]. We consider that a program would return an exact result if the computations were carried out using real numbers. In practice, roundoff errors arise during the execution and these errors are closely related to the way formulas are written. Our approach is based on abstract interpretation [5]. We build Abstract Program Equivalence Graphs (APEGs) to represent in polynomial size an exponential number of mathematically equivalent expressions [8]. APEGs are abstractions of the Equivalence Program Expression Graphs introduced in [14]. The concretization of an APEG yields expressions of very different shapes and accuracies. To synthesize expressions from APEGs, we use a profitability analysis which searches the most accurate expressions among all the expressions represented by the APEG.

This article focuses on our profitability analysis. An APEG is an abstraction of an exponential number of expressions and the profitability has to extract an accurate formula. We

$\dagger$ This work was partly supported by the SARDANES project from the french Aeronautic and Space National Foundation. compute safe error bounds using established static analysis techniques for numerical accuracy [10] and we use a limited depth search algorithm to explore the APEG structure. In addition, APEGs contain abstraction boxes representing any parsing of a sequence of operations defined by a set of operands and one commutative operator. We also define a way to synthesize an accurate formula from abstraction boxes.

For tractability reasons, we require our profitability analysis to be polynomial in the size of the APEGs. The APEGs representing an exponential number of expressions, our profitability is then a heuristic and we present experimental results to assert its efficiency. We implemented our techniques in a tool, named Sardana which takes as entry Lustre programs [4]. Inputs are represented by abstract streams which indicate, at each instant, a range for the values of the variables and a range for the roundoff errors on these variables. We present experimental results showing how our techniques improve the numerical formulas arising of several relevant pieces of codes extracted from an industrial critical software coming from aeronautics.

\section{APEG CONSTRUCTION}

In this section, we present our intermediate representation of programs, called Abstract Program Expansion Graph (APEG). This intermediate representation is inspired from the EPEGs introduced in [14]. However, as an EPEG is not necessarily complete, we define the APEGs as an intermediate structure between the initial PEGs and the theoretical complete EPEGs which can be intractable or infinite. The main objective of APEGs is to use abstractions in order to remain polynomial in size while still representing the largest number of equivalent expressions. APEGs contain a compact representation of many transformations of expressions in abstraction boxes which allow one to represent very large sets of expressions in polynomial size, despite that these expressions are of very different shape. An abstraction box is defined by a commutative binary operator, such as + or $\times$, and by a list of nodes which correspond to the operands. These nodes can be either constants, variable identifiers, sub-trees, equivalence classes or abstraction boxes. An abstraction box stands for all the parsings of the given leaves using the binary operator. For example,,$+(a, b, c, d)$ stands for all parsings of the sum $a+b+c+d$. Also,,$+\left(a, b, c, \times,\left(x_{1}, x_{2}, x_{3}\right)\right)$ stands for all the summations of the sum $a+b+c+X$, where $X$ stands for any parsings of the product $x_{1} \times x_{2} \times x_{3}$. So, an abstraction 


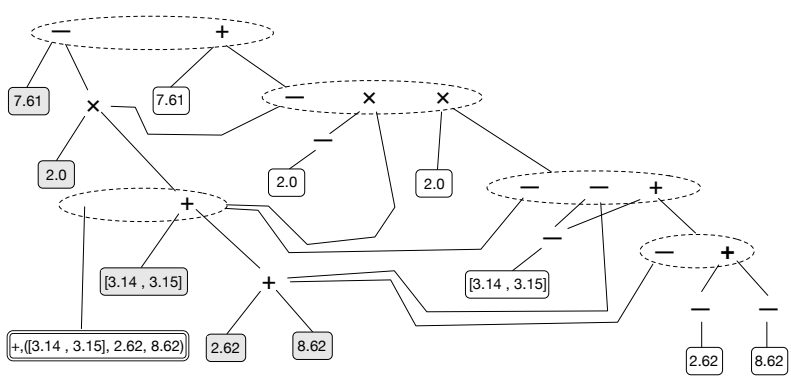

Fig. 1. Example of an APEG.

$$
\begin{aligned}
& \left(x_{1}^{\sharp}, \mu_{1}^{\sharp}\right)+\left(x_{2}^{\sharp}, \mu_{2}^{\sharp}\right)=\left(\uparrow_{0}^{\sharp}\left(x_{1}^{\sharp}+x_{2}^{\sharp}\right), \mu_{1}^{\sharp}+\mu_{2}^{\sharp}+l_{0}^{\sharp}\left(x_{1}^{\sharp}+x_{2}^{\sharp}\right)\right) \\
& \left(x_{1}^{\sharp}, \mu_{1}^{\sharp}\right)-\left(x_{2}^{\sharp}, \mu_{2}^{\sharp}\right)=\left(\uparrow_{0}^{\sharp}\left(x_{1}^{\sharp}-x_{2}^{\sharp}\right), \mu_{1}^{\sharp}-\mu_{2}^{\sharp}+l_{0}^{\sharp}\left(x_{1}^{\sharp}-x_{2}^{\sharp}\right)\right) \\
& \left(x_{1}^{\sharp}, \mu_{1}^{\sharp}\right) \times\left(x_{2}^{\sharp}, \mu_{2}^{\sharp}\right)= \\
& \quad\left(\uparrow_{0}^{\sharp}\left(x_{1}^{\sharp} \times x_{2}^{\sharp}\right), x_{1}^{\sharp} \times \mu_{2}^{\sharp}+x_{2}^{\sharp} \times \mu_{1}^{\sharp}+\mu_{1}^{\sharp} \times \mu_{2}^{\sharp}+\downarrow_{0}^{\sharp}\left(x_{1}^{\sharp} \times x_{2}^{\sharp}\right)\right)
\end{aligned}
$$

Fig. 2. Abstract semantics of the elementary operations for the floating-point arithmetic.

box is a very compact structure which is able to represent up to $1 \times 3 \times 5 \cdots \times 2 n-3$ possible evaluation schemes [12, $\S 6.3]$, where $n$ in the number of operands in the box.

APEG construction rely on two kinds of transformation algorithms: the propagation algorithms, and the expansion algorithms. Our approach consists of composing each of these algorithms together in order to produce the largest APEG, in the sense of the number of versions of a program it represents, while staying polynomial. We have designed several algorithms to add new shapes of expressions in an APEG. For example, we recursively propagate subtractions into the concerned operands, we propagate products, and we factorize common factors through the structure. Figure 1 represents an APEG, gray nodes and gray rectangles are the original PEG nodes, dashed circles are equivalence classes, the rectangle with a double outline on the left is an abstraction box. Except for the abstraction box present, this APEG illustrates how the propagation of the minus operator is done.

The expansion algorithms are designed to introduce abstraction boxes into the APEG. These algorithms search recursively in the APEG where a symmetric binary operator is repeated (we referred at these parts as homogeneous parts). When an expansion algorithm finds a homogeneous part it inserts a polynomial number of abstraction boxes into it, each of these abstraction boxes representing alternative versions of the homogeneous part. We have designed several polynomial algorithms to build APEG, described in [8]

\section{Profitability Analysis}

To compute safe bounds on the numerical accuracy of arithmetic expressions, we use abstract values $\left(x^{\sharp}, \mu^{\sharp}\right) \in \mathbb{E}^{\sharp}$ where $x^{\sharp}$ and $\mu^{\sharp}$ are intervals whose bounds are floatingpoint numbers, and where $x^{\sharp}$ represents the interval of values of the input and $\mu^{\sharp}$ represents the intervals of errors on the input [10]. A value $\left(x^{\sharp}, \mu^{\sharp}\right)$ abstracts a set of concrete values $\left\{\left(x_{i}, \mu_{i}\right), i \in I\right\}$ by intervals in a component-wise way.

For an arithmetic expression, the propagation of roundoff errors corresponds to the semantics of [10] and is given in Figure 2. The abstract function $\uparrow_{0}^{\sharp}$ corresponds to the concrete function $\uparrow_{0}$ which calculate the difference between a real value and its rounded floating-point value. We have:

$$
\uparrow_{0}^{\sharp}([\underline{x}, \bar{x}])=\left[\uparrow-\infty(\underline{x}), \uparrow_{+\infty}(\bar{x})\right]
$$

The function $\downarrow_{0}^{\sharp}$ is a safe abstraction of $\downarrow_{0}$, i.e. $\forall x \in[\underline{x}, \bar{x}], \downarrow_{0}$ $(x) \in \downarrow_{0}^{\sharp}([\underline{x}, \bar{x}])$. For example, if the current rounding mode $\circ$ is to the nearest, one may choose

$$
\downarrow_{0}^{\sharp}([\underline{x}, \bar{x}])=[-y, y] \quad \text { with } y=\frac{1}{2} \operatorname{ulp}(\max (|\underline{x}|,|\bar{x}|))
$$

where the $u$ nit in the last $p$ lace $\operatorname{ulp}(x)$ is the weight of the least significant digit of the floating-point number $x$ [13]. For an addition, the errors on the operands are added to the error due to the roundoff of the result. For a subtraction, the errors on the operands are subtracted. Finally, the semantics of the multiplication comes from the development of $\left(x_{1}^{\sharp}+\mu_{1}^{\sharp}\right) \times\left(x_{2}^{\sharp}+\mu_{2}^{\sharp}\right)$. The semantics of other operations is described in [10]. We use the former arithmetic for the elementary operations between floating-point values. In Lustre, values are streams and the operations have to be extended to streams.

As a synchronous language, Lustre [4] handles streams of values recording the values of a program point at each time instant. For example, let us consider the following program:

$$
\begin{aligned}
& \mathrm{a}=1.0 / 3.0 ; \\
& \mathrm{b}=1.0 \rightarrow \mathrm{a} \star \operatorname{pre}(\mathrm{b}) ;
\end{aligned}
$$

A constant stream mapping each instant to the internal representation of $\frac{1}{3}$ is associated to $a$. The stream $b$ is the stream whose value is $b(0)=1.0$ at time 0 and whose value at time $i$ is $b(i)=a(i) \times b(i-1)$.

Following the notations of [3], we denote $s=\left\langle m, t_{0}\right.$ : $\left.x_{0} \wedge t_{1}: x_{1} \wedge \ldots \wedge t_{N}: x_{N}\right\rangle$ the stream $s$ such that, at each instant $i \bmod m, s(i)=x_{k}$ if $t_{k} \leq i \bmod m<t_{k+1}$ for some $k \in[0, N-1]$ or $s(i)=x_{N}$ if $t_{N} \leq i \bmod m<m$. By extension, we consider that if $m=0$ then no modulo holds and $\forall i \geq t_{N}, s(i)=x_{N}$. We also assume that always $t_{0}=0$.

Our streams associate at each instant a value in the domain $\mathbb{E}$ of intervals of floating-point numbers with errors introduced in Section III. Hence, a stream $s \in \mathbb{S}$ is a mapping $s: \mathbb{N} \rightarrow \mathbb{E}$ where $\mathbb{N}$ denotes the set of non-negative integers. Coming back to our former example, the stream associated to $a$ is

$$
\begin{aligned}
\langle 0, \quad 0: \quad & ([3.33333333333325 E-1,3.33333333333334 E-1], \\
& {[1.85037170770855 E-17,1.85037170770861 E-17])\rangle }
\end{aligned}
$$

and the stream associated to $\mathrm{b}$ is

$$
\begin{array}{rll}
\langle 4, \quad 0: \quad & ([2.09075158128704 E-7,1.0], \\
& [-6.49870810424242 E-23,3.89955157246792 E-22]) \wedge \\
1: \quad & ([2.09075158128704 E-7,3.33333333333334 E-1], \\
& [-2.77556273678202 E-17,4.62596578069798 E-17]) \wedge \\
2: \quad & ([2.09075158128704 E-7,1.11111111111112 E-1], \\
& [-1.61907865481218 E-17,2.85268996889166 E-17]) \wedge \\
3: \quad & ([2.09075158128704 E-7,3.70370370370373 E-2], \\
& [-8.86638677409539 E-18,1.50345826165255 E-17])
\end{array}
$$

Elementary operations are applied at each instant, i.e if $s=$ $\left\langle m, 0: x_{0} \wedge t_{1}: x_{1} \wedge \ldots \wedge t_{N}: x_{N}\right\rangle$ and $s^{\prime}=\left\langle m^{\prime}, 0: x_{0}^{\prime} \wedge t_{1}^{\prime}:\right.$ 
$\left.x_{1}^{\prime} \wedge \ldots \wedge t_{N^{\prime}}^{\prime}: x_{N}^{\prime}\right\rangle$ then, for an operation $* \in\{+,-, \times\}$, we have $\left(s * s^{\prime}\right)(i \bmod k)=s(i \bmod m) * s^{\prime}\left(i \bmod m^{\prime}\right)$ where $k$ is the least common multiple of $m$ and $m^{\prime}$.

The profitability has to search an expression which minimizes the roundoff errors. As our values are element of $\mathbb{E}^{\sharp}$, we have to define in what sense we aim at minimizing the errors. First, let us introduce some notations. For a value $v=\left(x^{\sharp}, \mu^{\sharp}\right) \in \mathbb{E}^{\sharp}$, let $\mathcal{E}(v)=\mu^{\sharp}$. The function $\mathcal{E}$ gives the error term of an abstract value. Next, if $\mathcal{E}(v)=\mu^{\sharp}=[\mu, \bar{\mu}]$, then $M_{\mathcal{E}}^{+}(v)=\bar{\mu}, M_{\mathcal{E}}^{-}(v)=\underline{\mu}$, and $M_{\mathcal{E}}(v)=\max (|\underline{\mu}|,|\bar{\mu}|)$. In other words, $M_{\mathcal{E}}^{+}(v), M_{\mathcal{E}}^{-}(\bar{v})$ and $M_{\mathcal{E}}(v)$ denote the upper and lower bounds of the error and the maximal absolute error bound, respectively. We consider several orders:

- Strict order: $s \prec_{s} s^{\prime}$ if $\forall i \in \mathbb{N}, \mathcal{E}(s(i)) \subseteq \mathcal{E}\left(s^{\prime}(i)\right)$. This order requires that, if $s \prec_{s} s^{\prime}$ then at any time the error bound on $s$ is less than the error bound on $s^{\prime}$. The accuracy is improved at each instant.

- Max order: $s \prec_{m} s^{\prime}$ if $\max _{i \in \mathbb{N}} M_{\mathcal{E}}(s(i)) \leq M_{\mathcal{E}}\left(s^{\prime}(i)\right)$. This order only considers the worst errors, i.e. $s \prec_{m} s^{\prime}$ if the worst intensity of the error possibly associated to a value at a given instant is always smaller in $s$ than in $s^{\prime}$. Elsewhere, the errors may be greater in $s^{\prime}$ than in $s$.

- Integral order: $s \quad \prec_{i} \quad s^{\prime}$ if $\sum_{i=0}^{i=m-1} M_{\mathcal{E}}^{+}(s(i))-$ $M_{\mathcal{E}}^{-}(s(i)) \leq \sum_{i=0}^{i=m-1} M_{\mathcal{E}}^{+}\left(s^{\prime}(i)\right)-M_{\mathcal{E}}^{-}\left(s^{\prime}(i)\right)$. This order compares the integrals of the error functions $\mathcal{E}(s)$ and $\mathcal{E}\left(s^{\prime}\right)$. We have $s \prec_{i} s^{\prime}$ if the sums of the errors at each instant is smaller in $s$ than in $s^{\prime}$. If $s \prec_{i} s^{\prime}$ then at some instant $i$, the error $s(i)$ may be greater than the error $s^{\prime}(i)$. If $m=0$ or $m^{\prime}=0$, the integral is computed up to $\max \left(m, m^{\prime}\right)-1$. If $m=0$ and $m^{\prime}=0$, the integral is computed up to $\max \left(t_{N}, t_{N^{\prime}}^{\prime}\right)$.

The Strict order $\prec_{s}$ would require to synthesize a program which always improves the error bounds on the computed values. We consider that this order is too restrictive and we do not use it in practice. The Max order $\prec_{m}$ may be interesting in certain applicative contexts where the main objective is to lower the worst error bound and where the average error is not relevant. The Integral order $\prec_{i}$ gives a measure of the average error. We consider this order as the most interesting. In practice, our experiments confirm that the integral order is the order for which we may optimize the most the programs.

To synthesize an optimized program, the profitability has to be performed on the APEGs as defined in Section II. A main difficulty is that, thanks to equivalence classes, an APEG may represent an exponential number of expressions whose accuracy should be individually evaluated. To cope with this combinatorial explosion, we use a limited depth search strategy with memoization. We select the way an expression is evaluated by considering only the best way to evaluate its sub-expressions. This corresponds to a local choice.

Algorithm 1 illustrates how we perform the profitability when the depth is set to 1 . The operator :: appends a value to a list. It considers, for each node in the given equivalence class, the cartesian product of the elements of the node. It uses the equivalence classes of the node parameters and evaluates the

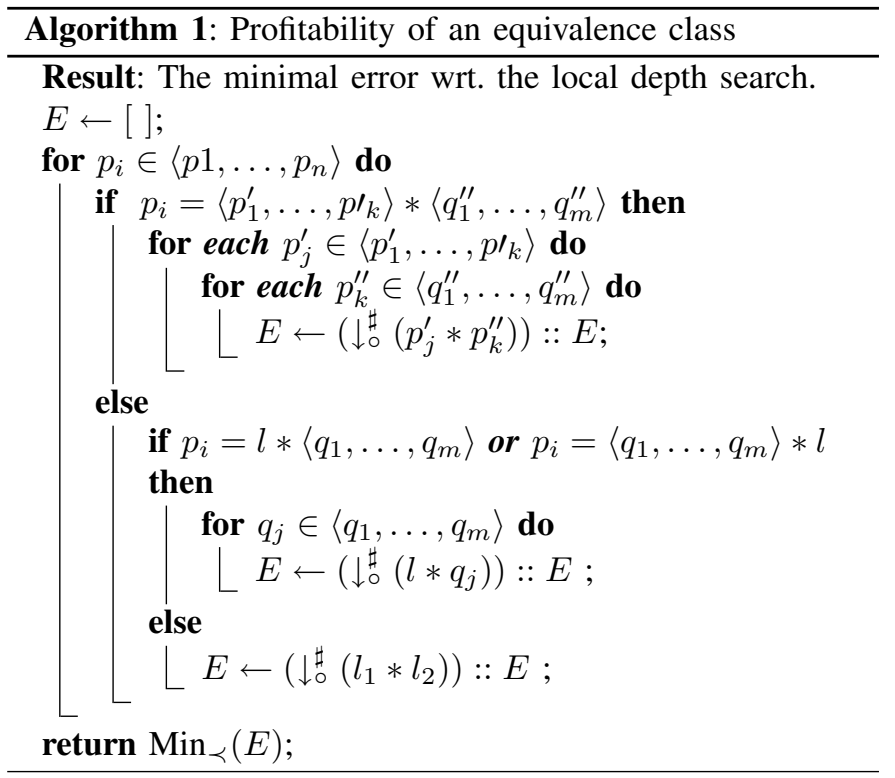

roundoff error generated by the operator using the semantics introduced above. Then it returns the minimal roundoff error for the desired order $\left(\prec_{s}, \prec_{m}\right.$ or $\left.\prec_{i}\right)$ after memoization of the accuracies of the expressions encountered. Algorithm 1 does not detail how to synthesize the final expression once we have found the minimal stream. This step is a simple propagation of the expressions along with the streams.

The next point concerns the synthesis of an expression for an abstraction box $B=*,\left(p_{1}, \ldots, p_{n}\right)$. In this case, we use an heuristic which generates an accurate expression (yet not always optimal). This heuristic is a greedy algorithm which searches at each step the pair $p_{i}$ and $p_{j}$ such that $\downarrow_{\circ}^{\sharp}\left(p_{i} * p_{j}\right)$ is minimal. Once it finds $p_{i}$ and $p_{j}$ it replaces both terms in the box by a new term $p_{i j}$ whose accuracy is equal to $\downarrow_{0}^{\sharp}\left(p_{i} * p_{j}\right)$. This heuristic computes in $O\left(n^{3}\right)$ iterations.

\section{CASE STUdY : AVIONIC BENCHMARKS}

We present in this section several experimental results obtained on pieces of code extracted from embedded critical industrial avionic codes. These experimental results concern in one hand programs using the IEEE-754 binary 32 format (Table I), and, on the other hand, programs using the IEEE-754 binary 64 format (Table II). For both floating-point formats, we have tested each program for many contexts, each having its own input values. Again, input values are described by means of streams of intervals of floating-point numbers. All the contexts we used have been kindly provided by the ASTRÉE team [2] who currently analyzes these programs and have access to realistic simulations of them. Note that even if some programs appear in both tables these programs have different codes and performances, yet they aim at achieving a similar task. In this case study, we use the Integral order. To perform our benchmarks, we used for each program and each context three identical scenarios in which Sardana computes streams of size 5,15 or 35 instants. For all the contexts of each program, 


\begin{tabular}{|c|c|c|c|c|c|}
\hline \multirow{2}{*}{ Program } & \multirow{2}{*}{ \#Contexts } & \multirow{2}{*}{$\%$ Opt } & \multicolumn{3}{|c|}{ \#Integral from } \\
\cline { 4 - 6 } & & & 0 to 5 & 0 to 15 & 0 to 35 \\
\hline Interpol & 2135 & $3.5 \%$ & $0.4 \%$ & $0.4 \%$ & $0.4 \%$ \\
\hline L-P filter 1 & 32 & $96.9 \%$ & $13.5 \%$ & $6.7 \%$ & $3.1 \%$ \\
L-P filter 2 & 501 & $57 \%$ & $6.6 \%$ & $3.1 \%$ & $1.3 \%$ \\
\hline H-P filter & 414 & $80.9 \%$ & $11.7 \%$ & $23 \%$ & $25.6 \%$ \\
\hline Transfer & 477 & $100 \%$ & $15.9 \%$ & $18.4 \%$ & $20.2 \%$ \\
\hline \multicolumn{3}{|c}{} & \multicolumn{4}{|c|}{$\%$ accuracy gain } \\
\hline
\end{tabular}

TABLE I

RESULTS ON PROGRAMS USING IEEE-754 BINARY 32 FORMAT.

\begin{tabular}{|c|c|c|c|c|c|}
\hline \multirow{2}{*}{ Program } & \multirow{2}{*}{ \#Contexts } & \multirow{2}{*}{$\% \mathrm{Opt}$} & \multicolumn{3}{|c|}{ \#Integral from } \\
\cline { 4 - 6 } & & & 0 to 5 & 0 to 15 & 0 to 35 \\
\hline Interpol & 7817 & $4.9 \%$ & $7.1 \%$ & $7.1 \%$ & $7.1 \%$ \\
\hline L-P filter 1 & 44 & $85,2 \%$ & $9,2 \%$ & $8,3 \%$ & $7 \%$ \\
L-P filter 2 & 618 & $50,3 \%$ & $7,5 \%$ & $5,2 \%$ & $4,1 \%$ \\
\hline H-P filter 1 & 42 & $61,9 \%$ & $14 \%$ & $9,5 \%$ & $2,5 \%$ \\
H-P filter 2 & 125 & $52 \%$ & $7,4 \%$ & $6,1 \%$ & $3,9 \%$ \\
\hline Transfer & 364 & $98,5 \%$ & $14,4 \%$ & $17,7 \%$ & $19,4 \%$ \\
\hline Sqrt & 76 & $80.2 \%$ & $6.3 \%$ & $6.3 \%$ & $6.3 \%$ \\
\hline
\end{tabular}

TABLE II

RESULTS ON PROGRAMS USING IEEE-754 BINARY 64 FORMAT.

we present in Tables I and II the improvement of the integral value of the errors for these scenarii. For industrial property reasons, we denote the programs used by the following generic terms: Interpol stands for a first order interpolation, $\mathrm{L}-\mathrm{P}$ filter are respectively a first order low-pass filter, $\mathrm{H}-\mathrm{P}$ filter is a first order high-pass filter, Transfer denotes a second order transfer function between two inputs, and Sqrt is a polynomial interpolation of the square root. We can draw two conclusions with these experiments. First, in many cases, the gain in accuracy decreases as the length of the streams increases. This can be observed on all low-pass filters described here. We explain this phenomenon by the fact that in most of these cases the values generated by the program tend towards zero and the errors on these values then decreases as well over time. Thus, the gain at each step of the integral tends to become smaller leading the integral gain to decrease as well. This conclusion is even more accurate to us, that in the other cases where the values are growing without limit (like with the high-pass filter in Table I or the Transfer program in Table II) the gain on the integral value increases as the stream length increases. The second conclusion is that our approach is able to improve the overall numerical accuracy of very different programs in many different contexts. For most programs this gain is between $2 \%$ and $20 \%$ for $50 \%$ to $90 \%$ of the contexts. For the few programs we are unable to improve such as Interpol in Table I, we may argue that these programs do not allow much syntactic transformations.

\section{CONClusion}

In this article we have presented a new profitability heuristic which allows us to extract a more accurate version of a program from our intermediate representation called APEG. This article briefly describes how APEGs are constructed, and how they are able to represent many equivalent versions of a program in order to find one with better numerical accuracy. We have designed a profitability analysis which runs in polynomial time recursively into the APEG and synthesize a well-formed, new, but yet mathematically equivalent, version of a program. Our experimental results show significant improvement for real case examples of industrial avionic code. We believe that our approach could be extended in many ways, we are confident that the profitability heuristic we currently use could be improve in order to synthesize even more accurate programs. Finally we consider that many other orders over the streams of values could be defined, and may improve the quality of the profitability heuristic we use.

\section{ACKNOWLEDGMENT}

We thanks Alexandre Chapoutot for his advises, Antoine Miné and all the ASTRÉE team for their contribution on the avionic benchmarks, also Laurent Thévenoux and Christophe Mouilleron for generating the evaluation schemes of expressions.

\section{REFERENCES}

[1] ANSI/IEEE. IEEE-754 Standard for Binary Floating-point Arithmetic, ed. 2008.

[2] J. Bertrane, P. and R. Cousot, J. Feret, L. Mauborgne, A. Miné, and $\mathrm{X}$. Rival. Static analysis and verification of aerospace software by abstract interpretation. In AIAA Infotech@Aerospace. American Inst. of Aeronautics and Astronautics, 2010.

[3] O. Bouissou and M. Matthieu. Abstract interpretation of the physical inputs of embedded programs. In VMCAI, pages 37-51, 2008.

[4] P. Caspi, D. Pilaud, N. Halbwachs, and J. Plaice. Lustre: A declarative language for programming synchronous systems. In POPL, pages 178 $188,1987$.

[5] P. and R. Cousot. Abstract interpretation: A unified lattice model for static analysis of programs by construction of approximations of fixed points. In Principles of Programming Languages 4, pages 238-252. ACM Press, 1977.

[6] D. Delmas, E. Goubault, S. Putot, J. Souyris, K. Tekkal, and F. Vedrine. Towards an industrial use of fluctuat on safety-critical avionics software. In Formal Methods for Industrial Critical Systems, 14th International Workshop, FMICS, 2009.

[7] E. Goubault and S. Putot. Static analysis of finite precision computations. In VMCAI'11, number 6538 in LNCS, pages 232-247, 2011.

[8] A. Ioualalen and M. Martel. A new abstract domain for the representation of mathematically equivalent expressions. In Static Analysis Symposium, SAS, number 7460 in LNCS, pages 75-93. Springer, 2012.

[9] A. Ioualalen and M. Martel. Synthesis of arithmetic expressions for the fixed-point arithmetic: The sardana approach. In The 6th Conference on Design \& Architectures for Signal \& Image Processing, DASIP, pages 346-353, 2012.

[10] M. Martel. Enhancing the implementation of mathematical formulas for fixed-point and floating-point arithmetics. Journal of Formal Methods in System Design, 35:265-278, 2009.

[11] D. Monniaux. The pitfalls of verifying floating-point computations. TOPLAS, 30(3), May 2008.

[12] C. Mouilleron. Efficient computation with structured matrices and arithmetic expressions. PhD thesis, Université de Lyon - ENS de Lyon, November 2011.

[13] J.-M. Muller. On the definition of ulp(x). Technical Report 5504, INRIA, 2005.

[14] R. Tate, M. Stepp, Z. Tatlock, and S. Lerner. Equality saturation: A new approach to optimization. Logical Methods in Computer Science, 7(1), 2011. 